\title{
Gated Communities \\ Ein Vergleich privatisierter Wohnsiedlungen in Südkalifornien
}

\author{
Markus Gmünder, Nathalie Grillon, Kuno Bucher, \\ Basel
}

\section{Einleitung}

Gated Communities bilden seit Ende der 1980er Jahre einen allgegenwärtigen Bestandteil der nordamerikanischen Siedlungslandschaft. Insbesondere in den Metropolitanräumen der Ost- und Westküste sowie im Sunbelt der USA ist eine Akkumulation dieser Wohnform festzustellen: Zu Beginn des 21. Jahrhunderts gibt es bereits über 205'000 privat organisierte Siedlungen (davon gut $10 \%$ Gated Communities) mit insgesamt über 42 Mio. (9 Mio. in Gated Communities) Einwohnern (Blakely \& SNyder 1997: 180, Treese 1999: 19).

Die Privatisierung des öffentlichen Raumes zeigt sich bei Gated Communities in ihrer ausgeprägtesten Form. Ziel dieses Aufsatzes ist es, die strukturelle Entwicklung solcher Siedlungen in ihrer Vielschichtigkeit aufzuzeigen und in einen räumlichen, sozialen und ökonomischen Zusammenhang zu stellen. Nach einer kurzen thematischen Einführung werden Erklärungsansätze für die Entstehung von Gated Communities erläutert und Fallbeispiele verschiedener Siedlungstypen kurz vorgestellt. Das Fazit bewertet im Kontext der international vergleichenden Stadtstrukturforschung deren Tragfähigkeit als künftige Wohnform und gibt mögliche Handlungsvorschläge an die Adresse der Planung.

\subsection{Raum- und Organisationsstruktur von Gated Com- munities}

Gated Communities umfassen Wohngebiete, deren Zugang beschränkt und in denen der öffentliche Raum (Strassen, Grünflächen etc.) privatisiert ist. Verschiedene Sicherheitseinrichtungen wie Mauern,Zäune und Wachhäuser mit Sicherheitspersonal erschweren das Betreten der Siedlung für Nichtbewohner (BLAKELY \& SNYDER 1997:2).

Die Kontrolle des Raumes wird durch eine entsprechende Gestaltung des Wohngebietes nachhaltig verändert. Newman (1997) unterscheidet in seinem Konzept des Defensible Space - ähnlich wie LichTENBERGER (1998) - zwischen privater, halbprivater, halböffentlicher und öffentlicher Interessensphäre und analysiert nebst dem Raum auch das Verhalten darin agierender Personen. Er folgert, dass sich der Einfluss einzelner Individuen auf den Raum in gleichem Masse reduziert, wie die Anzahl Personen, welche diesen Raum mitbenutzen, steigt. Obschon die amerikanische Siedlungsentwicklung nie einer zufälligen Bevölkerungsverteilung entsprach, weist der heutige Trend klar in Richtung vollständiger Vermarktung der Daseinsgrundfunktion Wohnen (LANGDON 1994: 73). Das Ergebnis ist eine Stadtlandschaft aus isolierten Landnutzungsfragmenten, deren räumliche Elemente konzeptionell voneinander abgegrenzte Kategorien bilden und klar definierte Aktionsräume für Wohnen, Arbeit und Freizeit schaffen. Diese Tendenz, Territorien um menschliche Aktivitäten zu bilden, manifestiert sich in den USA in Gated Communities, aber auch in den zahlreichen Country Clubs, Büro- oder Freizeitparks.

Eine Klassifikation der Gated Communites widerspiegelt bestenfalls eine Momentaufnahme der zum Zeitpunkt der Modellbildung bekannten Formen. Grundsätzlich können drei Typen von Gated Communities unterschieden werden: private Wohnsiedlungen, neu gebaute vollständige Städte (Gated New Towns) und sogenannte Security Zone Communities, entstanden durch die nachträgliche Umzäunung von Quartieren (WEHRHEIM 1999). Die nachfolgenden Ausführungen (siehe auch Grillon 1998, Bucher 1998, GMüNDER 1998) und Fallbeispiele beziehen sich, mit Ausnahme der Siedlung Leisure World, auf den ersten Typus, der je nach Zielgruppe (Rentner, Familien, Golfer etc.) eine andere Ausprägung hat.

Private umzäunte Gebietskörperschaften unterscheiden sich von herkömmlichen Siedlungsformen nicht nur bezüglich ihrer klaren physischen Abgrenzung nach aussen, sondern auch in ihrer internen Organisationsstruktur: Der Zusammenschluss gemeinsamer Interessen in einem privatisierten Gouvernanzsystem (Common Interest Development, CID) mit verbindlichen Kontrollvorkehrungen ermöglicht eine gezielte Steuerung der Siedlungsentwicklung. Die Homeowner Association (HOA) ist der Grundstein eines jeden Common Interest Development und hat die Aufgabe, den Unterhalt des Gemeinbesitzes, die allgemeine Wohnqualität sowie die Grundstückswerte zu sichern (vgl. Abb. 1). Die private Bereitstellung lokaler öffentlicher Güter (Ver-/Entsorgung, Freizeiteinrichtungen) wird von den Bewohnern über Gebühren abgegolten. Ähnlich Verwaltungsräten in der Privatwirtschaft übernimmt ein periodisch gewählter, ehrenamtlicher Vorstand (Board of Directors) die «Quasi-Exekutive» der HOA, welche die Interessen der Grundstückseigentümer zu vertreten hat. Vielfach werden alle administrativen Aufgaben externen Firmen 


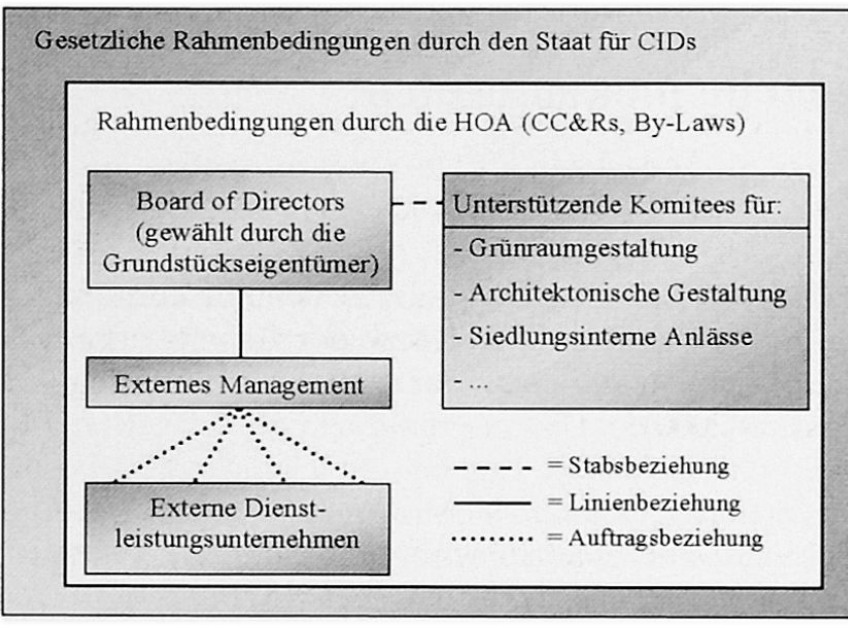

Abb.1: Organisationsstruktur von Gated Communities Administrative structure of Gated Communities Structure d'organisation des Gated Communities

anvertraut, die damit das gesamte operative Management der Siedlung übernehmen (DunBar 1988). Gated Communities stellen damit auch eine Extremform einer marktwirtschaftlichen, institutionellen Reform von Gemeinwesen dar.

Jeder Hauskauf in CIDs und somit auch Gated Communities ist an die Unterzeichnung von vertraglichen Klauseln, Bedingungen und Einschränkungen - sogenannte Covenants, Conditions and Restrictions (CC\&Rs) - gebunden. Diese beziehen sich sowohl auf Gemein- als auch auf Privatbesitz innerhalb der Siedlung und regeln die Rechte und Pflichten der Bewohner ebenso wie die der HOA. Sie ermöglichen ein hohes Kontrollniveau über bestimmte Standards und den Unterhalt der gesamten Siedlungsanlage (McKeNZIE 1994).

\section{Methodik und Erklärungsansätze zur Erfassung von Gated Communities}

In Anbetracht der rezenten Ausbreitungsdynamik von Gated Communities wäre eine quantitative Erfassung und Befragung der Bewohner zwar angemessen, in der Praxis jedoch kaum durchführbar, weswegen sich eine qualitative, vielschichtige Betrachtungsweise des räumlichen und gesellschaftspolitischen Umfeldes vor Ort aufdrängte. Im Rahmen eines Forschungsaufenthaltes in Südkalifornien im Herbst 1997 wurden von den Autoren diverse Experten- und Tiefeninterviews zur Gated Community-Thematik sowie Gruppendiskussionen mit direkt und indirekt Betroffenen durchgeführt. Mittels Feldbeobachtung sowie Kartierung von Siedlungsstrukturen konnte eine zielgerichtete, fallspezifische Untersuchung der ausgewählten
Beispiel-Kommunen vorgenommen werden (vgl. z.B. Atteslander 1995, Lamnek 1995, Patton 1990).

Für die Entstehung von Gated Communities stehen vier Erklärungsansätze im Vordergrund: (a) Sicherheit vor Kriminalität und deviantem Verhalten i.w.S., (b) Identifikation mit dem Wohnumfeld, (c) Staatsversagen und NIMBY-Problematik (Not In My BackYard), (d) Wahrung ökonomischer Interessen.

(a) Die Angst vor Verbrechen (Fear of Crime) ist in breiten Bevölkerungskreisen der amerikanischen Gesellschaft tief verwurzelt. Durch Medienberichte und Werbekampagnen negativ geprägte Wahrnehmungsmuster veranlassen viele Menschen, in Gated Communities Schutz vor Übergriffen durch Kriminelle und Zuflucht vor einem durch (sub-)kulturelles Verhalten anderer (z.B. Jugendkulturen) als negativ bewerteten Umfeld zu suchen. Dabei sind umzäunte und ummauerte Siedlungen das logische Ergebnis einer Entwicklung, die bereits mit dem Auszug der verunsicherten weissen Mittelklasse aus den Stadtzentren anfangs der 1950er Jahre eingesetzt hat (CHASE 1997). Angst vor Verbrechen ist nicht nur in Innenstädten, sondern auch in vergleichsweise sicheren Vororten eine treibende Kraft hinter dem Wachstum von Gated Communities.

(b) Die Identifikation mit dem Wohnumfeld ermöglicht den Bewohnern in Zeiten raschen sozialen und wirtschaftlichen Wandels, ihr persönliches Beziehungsnetz zu stabilisieren. Eine Gated Community dient dabei als klarer Bezugspunkt und Orientierungshilfe im Alltag. Die Community-Forschung sieht in der Gemeinschaft eine Kombination sozialer Einheiten und Systeme, die soziale Grundfunktionen mit lokaler Relevanz erfüllen (WARREN 1978: 9). Darunter fallen Produktion, Verteilung und Konsum von Gütern und Dienstleistungen sowie die Sozialisation bestimmter Verhaltensmuster. Mit der klaren räumlichen und sozialen Differenzierung durch Gated Communities versuchen Menschen in einer von Unsicherheit und gesellschaftlichem Wandel geprägten Zeit, Ordnung in eine unübersichtliche Siedlungslandschaft zu bringen: Sie entfliehen der sozialen Wirklichkeit in eine Welt, die gekennzeichnet ist durch ein Höchstmass an Privatsphäre.

(c) Das fehlende Vertrauen in das Handlungsvermögen staatlicher Behörden - hervorgerufen durch Staatsversagen - wird durch ein gesteigertes Kontrollbedürfnis über die persönliche Umgebung ersetzt (vgl. z.B. FoLDVARY 1994). Bei der Erstellung von Infrastruktur-Projekten (z.B. Flughafen, Autobahn oder Mülldeponie) an bestimmten Standorten divergieren die Interessen der lokalen Bevölkerung vielfach mit denjenigen der politischen Behörden, respektive der daran beteiligten 
Allgemeinheit. Die Opposition der Bewohner gegen derartige Vorhaben kommt im NIMBY-Syndrom und der Bildung lokaler Interessengemeinschaften zum Ausdruck (Frey 1997). Es beschreibt den organisierten Widerstand von Kommunen gegen unerwünschte exogene Einflüsse (Erstellung bestimmter Infrastrukturprojekte, Präsenz gewisser Bevölkerungsgruppen), der auch den Wunsch auf einen Verzicht institutionalisierter politischer Behörden widerspiegelt. In Gated Communities manifestiert sich der Wille, eine private Selbstverwaltung einzurichten, um ein allfälliges Staatsversagen zu umgehen, verbunden mit einem territorialen Verhalten, das die NIMBY-Problematik reflektiert.

(d) Die begrenzte Verfügbarkeit von Grundstücken in Gated Communities und die limitierte Zugänglichkeit siedlungsinterner Einrichtungen steigert den Investitionswert einer Kapitalanlage, die vor weiteren Wertverlusten durch interne Verwaltungs- und Entscheidungsstrukturen geschützt ist. Die private Bereitstellung lokaler öffentlicher Güter gewährleistet im Gegensatz zur staatlichen Erstellung auch eine Kostenanlastung nach dem fiskalischen Äquivalenzprinzip: Kostenträger, Nutzniesser und Entscheidungsträger sind dieselben Individuen (Olson, 1969). Dadurch können in Gated Communities gezielt bodenwerterhaltende und -steigernde Massnahmen umgesetzt werden. Dies kann durch eine sorgfältige Auswahl der zu erstellenden Kollektivgüter sowie durch die konsequente Vermeidung negativer externer Effekte geschehen. Das Interesse der Bewohner konzentriert sich dabei ganz auf den Erhalt der Grundstückswerte (Community Associations Institute Research FounDATION 2000).

\section{Vergleich verschiedener Gated Community-Typen}

Anhand der folgenden Fallbeispiele werden Entwicklungsmuster, räumliche Strukturen und Problemfelder unterschiedlicher Siedlungstypen erörtert (vgl. Abb. 2). Die vorgestellten Kommunen repräsentieren einen Ausschnitt der Gated Community-Landschaft im Metropolitanraum Los Angeles, einschliesslich angrenzender Gebiete wie dem Coachella Valley.

\subsection{Alterssiedlungen als Gated Communities}

Eine der ersten und erfolgreichsten Gated Community-Formen ist die Rentnersiedlung. Ältere Men-

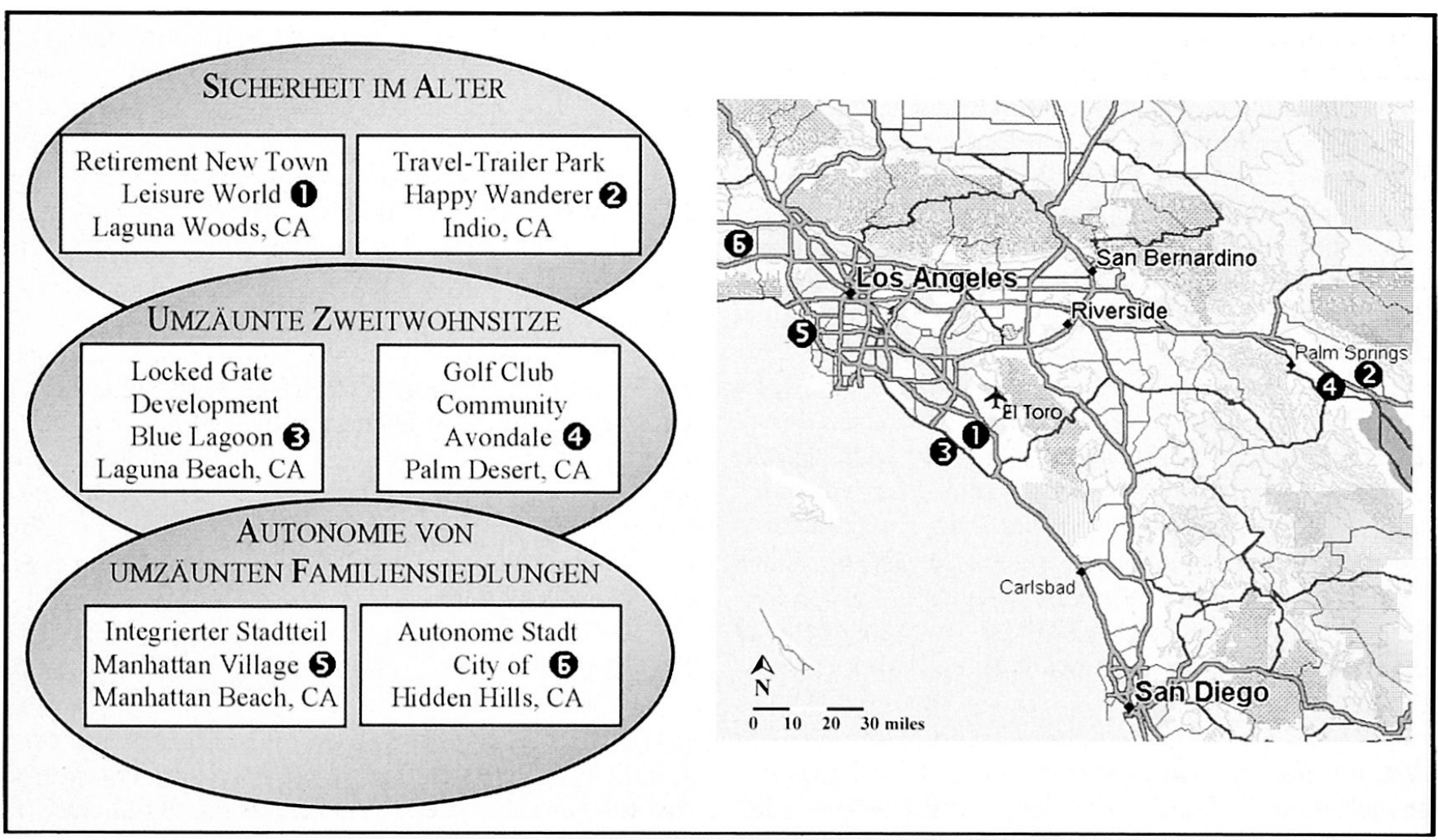

Abb. 2: Übersicht der untersuchten Siedlungen im Grossraum Südkalifornien

Overview of the investigated settlements in Southern California

Vue d'ensemble des zones d'habitat étudiées en Californie du Sud

(Kartengrundlage: eigener Entwurf nach DeLoRME MAP'N'Go) 
schen suchen in dieser Wohnform Schutz vor den als gefährlich erlebten urbanen Siedlungsräumen, aber auch sozialen Kontakt zu Altersgenossen. Stellvertretend für die zahllosen umzäunten Alterssiedlungen Südkaliforniens seien zwei Kommunen portraitiert, die aufgrund ihrer Verschiedenartigkeit die ganze Bandbreite dieser Entwicklung illustrieren.

Bereits im Jahre 1964 auf halber Strecke zwischen den beiden Metropolen Los Angeles und San Diego eröffnet, ist Leisure World Laguna Woods eine der ältesten und mit 18'000 Einwohnern gleichzeitig die grösste Gated Community für "Active Adults» in Südkalifornien (LW LaGUNA Woods 2000). Leisure World, seit 1999 politisch autonome Stadt (City of Laguna Woods), war jedoch nie nur Bauprojekt, sondern immer auch Vision eines völlig neuartigen Lebensstils. Das Siedlungskonzept dieser typischen Retirement New Town richtete sich von Beginn an primär auf aktive Senioren, die hier einen abwechslungsreichen, unbeschwerten dritten Lebensabschnitt verbringen wollen. Kernstück der Anlage ist daher konsequenterweise die aufwendige Freizeit-Infrastruktur, zu der u.a. sechs Klubhäuser, mehrere Golfplätze, ein privates Bustransportsystem, ein siedlungseigenes Fernsehstudio sowie zwei lokale Zeitungen zählen. Im Mittelpunkt der komplexen Organisationsstruktur stehen drei HOAs sowie die Golden Rain Foundation (GRF). Sie haben Verträge mit der auf Siedlungsmanagement spezialisierten Professional Community Management Inc. (PCM) abgeschlossen. PCM, die zweitgrösste "Senior Housing Management Firm» der USA, beschäftigt in Leisure World in den Bereichen Verwaltung/Finanzen, Sicherheit, Freizeit, Unterhalt, Landschaftsgestaltung und Transport ca. 1000 Personen (LW Laguna Woods 2000). Ein entscheidender Erfolgsfaktor dieser Seniorenstadt sind die umfangreichen Vorkehrungen zum Schutz vor Kriminalität. Die Investitionen für Mauern, Stacheldraht, bewachte Tore und eine private Polizeitruppe haben sich längst amortisiert, spielt doch der Sicherheitsaspekt beim Hauskauf im zunehmend dichtbesiedelten Orange County eine immer bedeutendere Rolle. Die Zufriedenheit mit dem Wohnumfeld äussert sich in der ausgeprägten Siedlungstreue vieler Bewohner (Aging in Place). Die Fluktuationsrate in Leisure World ist weitestgehend auf Todesfälle und nur selten auf einen Wohnortwechsel zurückzuführen.

Die Bevölkerungszusammensetzung des 1972 gegründeten Travel Trailer-Parks Happy Wanderer wird von mehreren Faktoren bestimmt: Aufgrund des Wüstenklimas im Coachella Valley bleiben während der Sommermonate einzig die knapp 40 Ganzjahresaufenthalter in der Anlage zurück, wogegen im Winter die Bevölkerungszahl durch das Eintreffen der sogenannten «Snowbirds» vor allem aus dem Mittleren
Westen und aus Kanada auf ca. 850 Personen zunimmt. Weiter ist Happy Wanderer aufgrund der CC\& Rs klar als Alterssiedlung («Adults 55 and over») konzipiert. Ähnlich wie im Fall von Leisure World Laguna Woods halten die meisten Bewohner der Siedlung über Jahrzehnte die Treue. Wer hierher zieht, hat sich ganz bewusst für eine nicht alltägliche Siedlungsform entschieden. Auch im Happy Wanderer RV Park wird aktive Freizeitgestaltung grossgeschrieben: Die Infrastruktur umfasst unter anderem zwei Klubhäuser mit Hobby- und Unterrichtsräumen, zwei Swimmingpools und diverse Sportanlagen. In zahlreichen Kleingruppen gehen die Senioren ihren Hobbies nach. Grundlage der Verwaltungsstruktur bilden die CC\&Rs und By-Laws. Erstere beschreiben die wichtigsten Rechte und Pflichten der Siedlungsbewohner sowie die Aufgaben der HOA, letztere definieren die rechtlichadministrative Organisation. Zentrales Element zur Bewahrung des Status und zur Gewährleistung stabiler Grundstückspreise sind auch in Happy Wanderer das Haupttor und die Umfassungsmauern (vgl. Abb. 3). Erst 1982 errichtet, prägen sie heute das Siedlungsbild und repräsentieren gleichzeitig die räumliche Abschottung nach aussen, was sogar im Gated Community-durchsetzten Südkalifornien bei Travel TrailerSiedlungen die Ausnahme darstellt.

Sicherheit im Alter ist ein Thema, welches Kriminalistik, Soziologie und Gerontologie seit Jahren gleichermassen beschäftigt. Verschiedene Studien haben nachgewiesen, dass Menschen mit zunehmendem Alter eine immer ausgeprägtere Furcht vor Verbrechen (Fear of Crime) entwickeln. Die Untersuchungsergebnisse lassen auf eine überdurchschnittliche Zunahme in der Altersgruppe über 65 schliessen, in der 86 Pro-

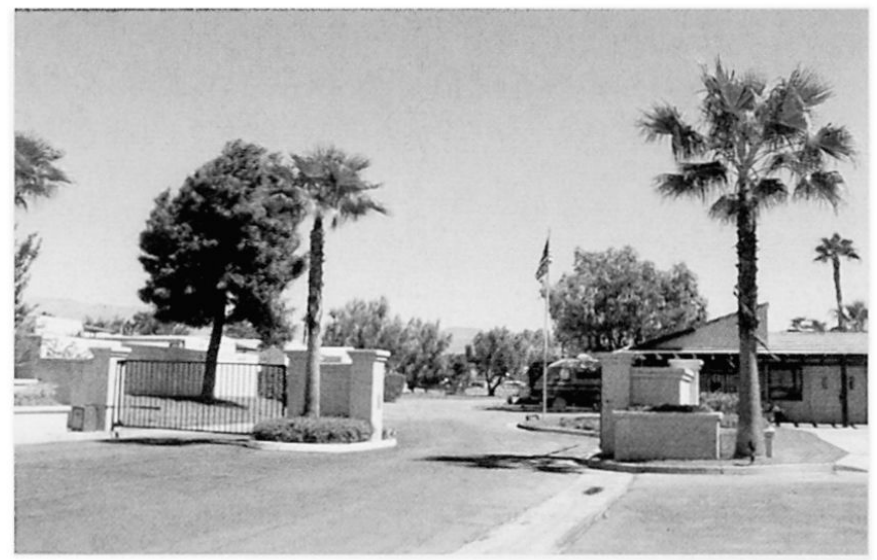

Abb. 3: Happy Wanderer Travel-Trailer Park: Haupttor Happy Wanderer Travel-Trailer Park: main gate Happy Wanderer Travel-Trailer Park: entrée principale (Photo: N. Grillon) 
zent der Ansicht sind, die Kriminalität habe seit ihrer Jugend überproportional zugenommen (VIERCK 1990: 84). Angesichts dieser Tatsache ist der Wunsch vieler Senioren nach einem sicheren, überwachten Wohnumfeld nicht weiter erstaunlich. Sie sehnen sich nach Siedlungen, in denen sie sich auch nachts gefahrlos bewegen können, und nach Nachbarn, auf die im Notfall Verlass ist (WeKerLe \& WhITZMan 1995). Alterssegregierte und ummauerte Kommunen wie Leisure World und Happy Wanderer sind als Antwort des Immobilienmarktes auf dieses Bedürfnis zu verstehen. Hier schaffen Tore, strenge Verhaltensregeln, Neighborhood Watch-Programme, gepflegte Gartenanlagen und saubere Strassen sowie aufmerksame Bewohner «Defensible Spaces», in denen sich Betagte geborgen fühlen (Dychtwald \& Flower 1990: 135). Doch die Wirksamkeit von Barrieren und Patrouillenfahrten im Kampf gegen die Kriminalität wird sich wohl nie exakt messen lassen. So kann die trotz vereinzelter Einbrüche sehr niedrige Verbrechensrate von Leisure World Laguna Woods - die Rentnerstadt ist gemäss Statistik eine der sichersten Gegenden des gesamten Bezirks (HUNT 1983: 36) - nicht nur darauf zurückgeführt werden, dass es sich bei der Kommune um eine Gated Community handelt. Andere siedlungsinterne (z.B. Altersstruktur, Bevölkerungszusammensetzung und Sozialstatus der Bewohner) und -externe Faktoren (z.B. generell niedrige Verbrechensrate im südlichen Orange County) spielen eine ebenso grosse Rolle. Grundsätzlich ist die Errichtung von Mauern und Toren nicht nur eine wirkungsvolle Sicherheitsmassnahme, sondern eine sehr erfolgreiche Marketingstrategie getreu dem Motto: «Security sells»!

\subsection{Gated Communities als Zweitwohnsitze}

Seit dem vermehrten Aufkommen privater Sports \& Country Clubs Mitte der 50er Jahre erfreuen sich Gated Communities als Anlagen für Wochenendaufenthalte und als Zweitwohnsitze grosser Beliebtheit. Die erfolgreiche Vermarktung des «Resort-Lifestyle» kommt vor allem in küstennahen oder golforientierten Privatsiedlungen in Florida und Südkalifornien zum Ausdruck. Die folgenden Kurzportraits sollen dies exemplarisch veranschaulichen.

Blue Lagoon ist ein klassischer Vertreter für die in südkalifornischen Küstenstädten häufig anzutreffenden «Locked Gate Developments» - ein Vorläufer der Gated Community, der das für den Küstenbereich typische traditionelle Einzelhausparzellenmuster durchbricht und ein Wohnviertel nach aussen abschottet, um den Strandzugang zu kontrollieren (vgl. Abb. 4). Das zwei Hektaren grosse Siedlungsareal umfasst 15 Gebäude mit 119 Apartments, zwei Poolanlagen, Gemeinschaftsräume und einen Tennisplatz. Um mehr Freiraum für Gemeinschaftseinrichtungen zu schaffen, wurde das Konzept eines Planned Unit Devlop-

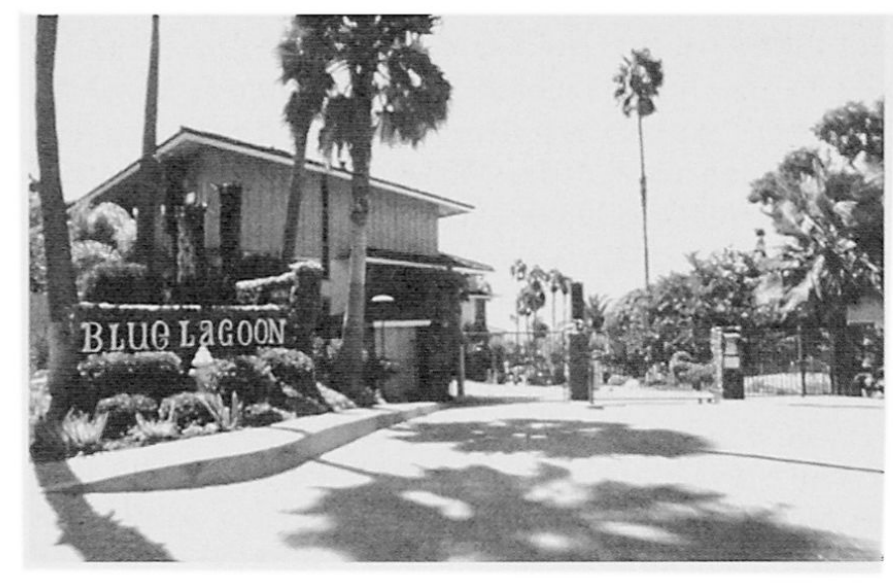

Abb. 4: Blue Lagoon: Eingangsbereich

Blue Lagoon: entrance area

Blue Lagoon: aire d'entrée

(Photo: N. GriLlon)

ment (PUD) verwendet, das die Erhöhung der Wohndichte in bestimmten Bereichen erlaubt. Diese Form des Cluster Zoning stellt den gesetzlichen Rahmen zur Verfügung, um via $\mathrm{HOA}$ den Erhalt der Bausubstanz (Unterhalt der internen Infrastruktur über Reserve-Fonds) und eine sichere Investitionsbasis für die weitere Siedlungsentwicklung gewährleisten zu können (Cullingworth 1993: 52ff.). Seit der Siedlungsgründung im Jahre 1964 sind die Apartmentpreise von 10'000 auf 500'000 USD bzw. 1 Million USD pro Einheit und je nach Lage angestiegen. Mit zunehmendem Marktwert der Grundstücke in Küstennähe erhöht sich der Druck, ältere Siedlungsstrukturen im Küstenbereich durch neue, marktgerechte Objekte zu ersetzen. Die verschärfte Situation auf dem Wohnungsmarkt hat auch in Blue Lagoon dazu beigetragen, dass sich immer weniger Familien die Wohnsiedlung am Privatstrand leisten können. Von den 300 Personen, die ein Apartment in Blue Lagoon besitzen, wohnen nur gerade 30 bis 40 das ganze Jahr dort. Viele sind schon im Ruhestand und wohnen seit der Siedlungsgründung da. Die Kombination von Privatstrand, exklusiver Küstenlage, Unterhalt des Gemeinbesitzes und Absicherung gegen unerwünschte Besucher machen Blue Lagoon zum begehrten Zweitwohnsitz wohlhabender Geschäftsleute oder Prominenter aus Los Angeles.

Avondale ist mit ihren 22 Jahren die älteste golforientierte Gated Community von Palm Desert. Sie bestand ursprünglich aus einem öffentlichen Golfplatz und einer privaten Gated Community und besass zudem ihre eigene Wasserquelle, womit die Grundlage für die entstehende Golf- und Gartenlandschaft gesichert war. Erst mit der vollständigen Erschliessung im Jahre 1984 kam es zum Zusammenschluss und zur eigentlichen Gründung von Avondale in der heutigen Form: 
der mittlerweile privatisierte Golfklub wurde vollständig in die nun 145 Hektaren umfassende Siedlung integriert. Ausschlaggebend war die Abwälzung der gestiegenen Kosten der Sicherheitseinrichtungen auf alle Nutzniesser. Da von den 290 Parzellen 45 noch unbebaut sind, verändert sich das Siedlungsbild kontinuierlich. Neben 74 Zweifamilienhäusern im Eingangsbereich gibt es 170 freistehende Villen in unterschiedlichster Form und Grösse (Parzellen von 900 - $7000 \mathrm{~m}^{2}$ ). Dementsprechend breit gestreut sind die Preise, von 150'000 USD bis weit über zwei Millionen. Rund ein Viertel der Objekte ist derzeit permanent bewohnt. Die Mitgliedschaft in der HOA allein berechtigt noch nicht zur Mitbenutzung der Golfanlagen und Klubeinrichtungen, da der Golfklub unabhängig von der Wohnsiedlung verwaltet wird. Nicht-Golfer können gegen eine geringe monatliche Gebühr als Social Member das Restaurant, die Spieltische und Tennisplätze mitbenutzen. Sowohl die Mitglieder des Golfklubs als auch die Bewohner profitieren gleichermassen von der zentralen Lage im Coachella Valley und der abgeschirmten, privaten Atmosphäre unter Gleichgesinnten in einer rund-um-die-Uhr bewachten Gated Community.

Auf dem lebensstilorientierten Wohnungsmarkt hat die Freizeitgestaltung einen hohen Stellenwert, und mit der Nachfrage nach immer neuen Trends ist in dieser Hinsicht auch kein Rückgang dieser Entwicklung abzusehen. Einzig die Verfügbarkeit geeigneter Standorte kann dem Boom einen Dämpfer versetzen. Umzäunte Siedlungen mit Privatstränden sind nach wie vor sehr beliebt, doch ihre Zahl wird in den meisten Küstenstaaten bereits gesetzlich limitiert und neue Anlagen werden zumindest auf das Privileg eines Privatstrandes verzichten müssen (KILDOw 1997). Der Wettbewerb zwischen privaten Freizeitumgebungen schraubt die Anforderungen betreffend Prestige, Exklusivität und Sicherheit immer höher: Hier bieten Gated Communities die entsprechende Organisationsstruktur, um Freizeiträume und deren Nutzungskapazität auf privatwirtschaftlicher Basis optimal und interessengerecht $\mathrm{zu}$ verwalten. Als Anlageobjekte können Zweitresidenzen in umzäunten Privatsiedlungen wie Blue Lagoon eine interessante Investition mit langfristiger Wertsteigerung sein. Im Zuge einer allfälligen Umnutzung zum Festwohnsitz stellt sich allerdings die Frage, ob die veränderten Anspruchsmuster immer noch abgedeckt werden können, insbesondere bei hohen Konzentrationen privater Wohnviertel wie im Coachella Valley, wo sich die Stadtverwaltungen mit einer steigenden Inanspruchnahme zentralörtlicher Einrichtungen und Dienstleistungen konfrontiert sehen. Hierbei tritt auch die Problematik zwischen öffentlichem und privatem Raum offen zu Tage: Mit der zunehmenden Privatisierung öffentlicher Erholungsgebiete und dem Vormarsch exklusiver Nut- zungsrechte durch Gated Communities wächst der Druck auf die lokale Planungspolitik, einen tragfähigen Konsens zwischen der Erhaltung alter Privilegien (Wahrung der Privatsphäre der Bewohner, die wie im Fall von Blue Lagoon den Strand einschliesst) und der Freihaltung gesetzlich geschützter Ausgleichsflächen bzw. Reintegration privater Nutzungsmuster in öffentliche Siedlungsstrukturen zu finden (vgl. z.B. LicHTENBERGER 1999: 37).

\subsection{Autonomie von Familienkommunen}

Der Autonomiegrad von Gated Communities ist unterschiedlich stark ausgeprägt. Unter diesem Aspekt wird nachfolgend anhand zweier, auf die Zielgruppe Familien ausgerichteter Gated Communities die Ausgestaltung des unterschiedlichen politischen Status dargestellt.

Wie die meisten Gated Communities liegt auch die 1984 erbaute private Familiensiedlung Manhattan Village innerhalb der Verwaltungshoheit einer offiziellen Stadt. Ihr Standort ist Manhattan Beach, eine südlich des Los Angeles International Airport gelegene Beachtown unweit regionaler Arbeitsplatzzentren mit guter Verkehrsanbindung. Die Siedlung hat rund 1600 Einwohner und ist vom Konzept her - ebenso wie Blue Lagoon - ein PUD, was auch die hohe bauliche Dichte von 21.5 Wohnheiten pro Hektar erklärt. Mit drei verschiedenen Haustypen vom freistehenden Einfamilienhaus bis zu den aneinandergebauten, dreistöckigen Courthomes mit mehreren Apartments - sowie zwei Poollandschaften, grosszügigen Grünanlagen und einem Kinderspielplatz wird versucht, den Ansprüchen junger Familien gerecht zu werden. Zudem profitiert Manhattan Village auch von der Nähe zu den einen guten Ruf geniessenden Bildungseinrichtungen des Manhattan Beach School-Districts (COHEN-Edwards 2000). Zwei HOAs, unterstützt durch mehrere freiwillige Komitees, dienen der Interessenvertretung aller Grundstücksbesitzer und der Aufrechterhaltung des hohen Siedlungsstandards. Während sich die Beziehungen der Bewohner zu ihrer Umgebung, d.h. Manhattan Beach und ihren Einrichtungen im Alltag als unkompliziert erweisen, sind die Kontakte zwischen der HOA und der Stadtverwaltung teilweise problematisch. Insbesondere die Umzäunung und die beschränkten Zutrittsmöglichkeiten sorgten zu Beginn für ein angespanntes Verhältnis zwischen der Siedlung und den öffentlichen Behörden. Als Vertretung finanzkräftiger Steuerzahler konnte die HOA jedoch ihre Interessen gegenüber der Stadt geltend machen und die Errichtung der Mauern und Tore durchsetzen.

Die im Nordwesten von Los Angeles im San Fernando Valley gelegene Gated Community Hidden Hills ist eine der wenigen privaten Kommunen, die über den 
autonomen Status einer offiziellen Stadt verfügen. Die Siedlung mit ihren knapp 2000 Einwohnern ist nach der Typologie von BanHam (1987) eine typische Vertreterin der Foothill Estates; charakteristisch ist auch die gemäss CC\&Rs vorgeschriebene geringe Dichte von nur gerade 2.5 Wohneinheiten pro Hektar. Die räumliche Struktur unterstützt damit das Bild einer familiären Small Town, die zugleich exklusives Refugium für erfolgreiche Unternehmer und Persönlichkeiten aus der Unterhaltungsindustrie sein soll. Die 1950 gegründete Siedlung bietet, nebst dem ländlichen Erscheinungsbild sowie den üblichen Freizeiteinrichtungen (Poolanlage, Tennisplätze etc.), eine spezifische Infrastruktur für «Pferdeliebhaber» mit grosszügig angelegten Reitwegen und spezialisiert sich somit innerhalb des Gated Communities-Marktes. Die Abgeltung dieser privat erstellten Kollektivgüter erfolgt direkt über eine damit verbundene Bodenwertsteuer. Als autonome Stadt und Gated Community verfügt Hidden Hills mit der HOA und einem offiziellen Stadtrat über zwei unterschiedliche Institutionen zur Verwaltung der Siedlung: Die HOA ist in erster Linie für interne Angelegenheiten (bspw. Infrastruktur, Administration etc.) zuständig, während der City Council die Siedlung nach aussen vetritt und für die Einhaltung der Vorgaben seitens der Bezirksbehörden zu sorgen hat (vgl. Abb. 5). Der Betrieb der auf dem Territorium der Stadt gelegenen Grundschule obliegt dem L.A. County. Aufgrund der umfassenden Einrichtungen spielt sich ein Grossteil des sozialen Lebens innerhalb der Siedlung ab. Auch auf institutioneller Ebene halten sich die Beziehungen zur Umgebung nicht zuletzt aufgrund der Autonomie in Grenzen; die Zusammenarbeit mit angrenzenden Städten oder dem L.A. County beschränkt sich auf regionale Infrastruktur- oder Planungsfragen (GlionNA 1994).

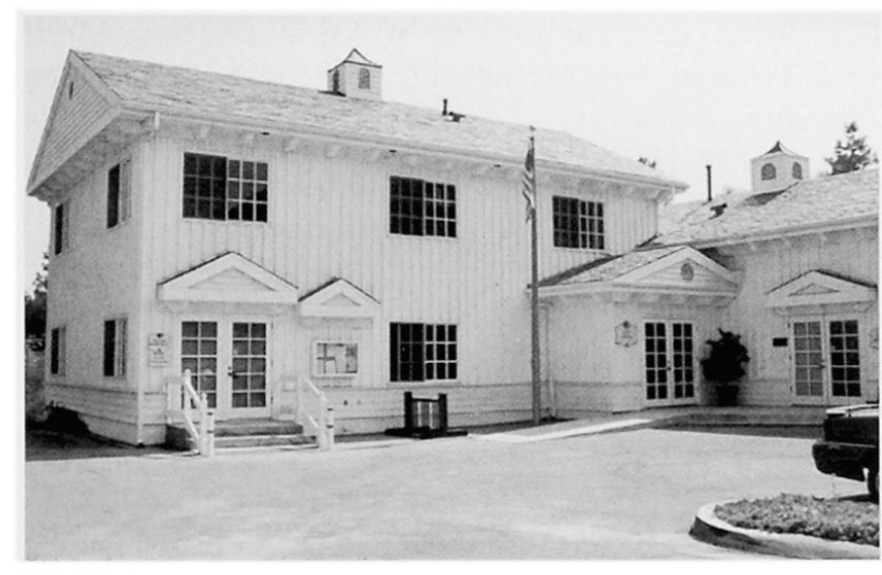

Abb. 5: Hidden Hills: Verwaltungsgebäude

Hidden Hills: city hall

Hidden Hills: mairie

(Photo: N. GRILLON)
$\mathrm{Zu}$ Beginn des 21. Jahrhunderts existieren in den USA gerade nur sieben Gated Communities, welche politische Autonomie geniessen. Doch das Potenzial für weitere politisch autonome Gated Communities ist gegeben, womit eine Ausweitung der Interessensphären dieser Siedlungen möglich ist. Die Homeowner Associations - auch Shadow Governments genannt - haben sich dabei an den Präferenzen der Grundstückseigentümer und somit am Erhalt der Grundstückswerte zu orientieren. Demokratische Rechte werden teilweise durch Marktbeziehungen respektive Konsumentenrechte ersetzt (SchaltegGer \& GmüNDER 1999). Die Interessenwahrnehmung erfordert jedoch auch eine Partizipation der Bewohner bei der Bewältigung siedlungsinterner Aufgaben. Aber gerade HOAs kleinerer oder neuerer Siedlungen leiden unter der mangelnden Bereitschaft, Aufgaben zu übernehmen; ein Sachverhalt, der auch in Manhattan Village zu beobachten ist. Alexander (1994) sieht den Grund dafür in den oftmals komplizierten Strukturen und Regelungen der HOAs. Fehlende Zeit oder zu geringe Anreize bei der Übernahme ehrenamtlicher Aufgaben dürften insbesondere bei Familiensiedlungen mit einer hohen Quote an Erwerbstätigen weitere Gründe sein. Anders verhält sich die Situation in etablierten Gated Communities wie Hidden Hills oder Rentnersiedlungen; dort ist die Passivität weitaus weniger ausgeprägt. Anreize wie der Nutzen in Form von sozialem Ansehen geben oftmals den Ausschlag für das Engagement bei siedlungsinternen Aufgaben und damit der Interessenvertretung der Bewohner.

\section{4 Überprüfung der Erklärungsansätze anhand der ausgewählten Kommunen}

Die Analyse der sechs ausgewählten Gated Communities hat die Angst vor Verbrechen als einen der Hauptgründe für das Leben in einer ummauerten Kommune bestätigt. Wie die Gespräche mit Bewohnern, Siedlungsmanagern, Grundstücksmaklern und anderen Betroffenen zeigen, symbolisieren Mauern, Zäune und Tore für Menschen vor allem physische Sicherheit. In einer Gesellschaft, in der die Gräben zwischen den sozio-ökonomischen Schichten spürbar tiefer geworden sind, lassen sich die Erfolgreichen den Schutz ihres Eigentums immer mehr kosten. Der Stellenwert, welcher dem Sicherheitsaspekt beim Kauf einer Immobilie in einer Gated Community zukommt, kann zwischen den verschiedenen Typen privater Siedlungen variieren. Zwar hat der Schutz von Eigentum in jedem Fall Priorität, doch scheinen die Bewohner von Anlagen mit Zweitwohnsitzen sowie von Alterskommunen diesem Faktor überdurchschnittlich viel Gewicht beizumessen. Oft wurden in den Interviews auch andere Faktoren wie das umfassende Dienstleistungs- und Sportangebot in den Vordergrund gestellt. 
Der Erfolg der Sicherheitsmassnahmen darf nicht darüber hinwegtäuschen, dass abgeschlossene Siedlungen die Angst vor Verbrechen noch verstärken können.

Nach Ansicht der Bauunternehmer (Developer) erlauben Gated Communities den Bewohnern, eine Abgrenzung nach aussen aufzubauen und den Zutritt zu ihrem Territorium zu kontrollieren, um auf diese Weise ein Identitäts- und Sicherheitsgefühl zu entwickeln. Das Wissen um gefestigte physische Abgrenzungsformen erleichtert die Steuerung sozialer Interaktion, reduziert das siedlungsinterne Konfliktpotenzial und fördert die Identifikation mit der Wohnumgebung und den Mitbewohnern. Erfahrungsgemäss ist der Gemeinschaftsgedanke in Gated Communities nicht mehr und nicht weniger verankert als in anderen Siedlungen auch (BLAKELY \& SNYDER 1997). In den beiden alterssegregierten Kommunen ist die Verwurzelung der Bewohner in ihrem Wohnumfeld deutlich zu spüren, während dies in den Familienkommunen stark von Alter und Struktur der Siedlung abhängt. So ist beispielsweise in Hidden Hills ein wesentlich stärkeres Identifikationsempfinden mit der Siedlung festzustellen als in Manhattan Village. Nicht überraschend identifizieren sich Bewohner weniger stark mit Zweitwohnsitzen als mit ihrem Hauptwohnort. Kommt es aber zu ausgedehnten Aufenthalten von einem halben Jahr oder mehr, wie in vielen Golfkommunen üblich, so ist die Ortsbindung - vor allem wenn ein bestimmter Lebensstil und entsprechende Sozialstrukturen (Klubmitgliedschaft) vermittelt werden - stärker ausgeprägt.

Grundsätzlich unterscheiden sich viele der Aufgaben und Funktionen, welche von Gated Communities wahrgenommen werden, nur unwesentlich von denjenigen herkömmlicher Städte und Kommunen. Die Annahme, dass die private Selbstverwaltung in Gated Communities eine Präventivmassnahme gegenüber einem potenziellen Staatsversagen darstellt, hat sich im Rahmen der in dieser Studie durchgeführten Expertengespräche und Interviews mit Betroffenen weitestgehend bestätigt. Hinweise auf die NIMBYProblematik sind vor allem bei Leisure World Laguna Woods erkennbar: Die Umwandlung des nahe gelegenen Luftwaffenstützpunktes El Toro in einen zivilen Flughafen mit einer Kapazität von 24 Millionen Passagieren führte zu heftigem Widerstand seitens der Siedlung (HaLdane 1998).

Verglichen mit konventionellen Siedlungen erlauben Gated Communities den Einsatz bestimmter werterhaltender Massnahmen, beispielsweise durch eine sorgfältige Auswahl der zu erstellenden lokalen öffentlichen Güter. In allen sechs Fallbeispielen besteht allgemein ein grosses Interesse am Erhalt, respektive der
Steigerung der Bodenwerte. Insbesondere in den Siedlungen Avondale, Blue Lagoon, Hidden Hills und Manhattan Village mit ihrer beachtlichen Bodenpreisentwicklung scheint diese Präferenz besonders ausgeprägt zu sein. Die Tore dieser Kommunen garantieren die exklusive Nutzung der lokalen öffentlichen Güter, und die CC\&Rs sorgen für ein geordnetes Erscheinungsbild, von dem letztlich alle Bewohner profitieren.

\section{Fazit und Ausblick}

Für immer mehr Amerikaner stellen Gated Communities und privat organisierte Siedlungen - sei es als Altersresidenz, als Zweitwohnsitz oder als kinderfreundliche Bleibe - das ideale Wohnumfeld dar, welches Sicherheit vermittelt und die Kontrolle über die unmittelbare Umgebung zu garantieren scheint. Private Enklaven gewinnen zunehmend auch ausserhalb der klassischen Verbreitungsgebiete in Südkalifornien, Florida und Texas an Popularität und sind weit mehr als nur eine Antwort auf tiefgreifende strukturelle Veränderungen der Wirtschaftsweise und des Zusammenlebens. Für die Bauwirtschaft bzw. die Developer bieten sie die Möglichkeit, eine gezielte Produktedifferenzierung zu betreiben und auf dem Haus- und Wohnungsmarkt aktiv neue Nischen zu erschliessen. Gated Communities sind mittlerweile für einen Grossteil der amerikanischen Bevölkerung zu einem erstrebenswerten Statussymbol und zur Verkörperung des idealen US-amerikanischen Lebensstils geworden.

\subsection{Vor- und Nachteile privatisierter umzäunter Wohn- gebiete}

Die bedeutendsten Vorteile von Gated Communities liegen bei ihrer marktwirtschaftlich orientierten Struktur. Die klare Zuteilung der Eigentumsrechte, verbunden mit der Ausrichtung an den Präferenzen der Bewohner sowie einer verbesserten fiskalischen Äquivalenz sind Gründe, die für den Erfolg dieser Form von Gemeinwesen sprechen. Die Marktprozesse verhindern im Vergleich zu konventionellen Kommunen ein Staatsversagen weitgehend. Diese Vorteile treffen jedoch nicht nur auf Gated Communities zu, sondern auf sämtliche privat organisierte Gebietskörperschaften oder CIDs.

Demgegenüber birgt aber das ungebrochene Wachstum dieser in jeder Hinsicht exklusiven Siedlungsstruktur auch verschiedenartige Probleme. So ist beispielsweise hinter die komplexen und bisweilen starren CC\&Rs ein Fragezeichen zu setzen, da zeitgerechte Strukturanpassungen erschwert werden. Um in 20 bis 30 Jahren neuen gesellschaftlichen Bedürfnissen wie veränderten Haushaltsgrössen und -zusammensetzungen gerecht zu werden, müssten die CC\& Rs möglichst flexibel und für die Mehrheit der Bewohner nachvollziehbar gestaltet werden (MCKENZIE 1994). 
Gated Communities leisten in einer an sich schon stark segregierten Kulturlandschaft der Ghettoisierung weiter Vorschub. Dabei handelt es sich in erster Linie um eine Fragmentierung nach Lebensstilen bzw. sozioökonomischen Kriterien und weniger nach ethnischen Merkmalen. Somit stellen Gated Communities eine zusätzliche Möglichkeit zur Verfügung, um sich einen individuell bestimmten privaten Lebensraum ausserhalb öffentlich zugänglicher Interaktionsräume zu schaffen und die Beziehungen nach aussen auf ein Minimum zu reduzieren. Dies birgt die Gefahr einer verzerrten Wahrnehmung der räumlichen und sozialen Umwelt, verbunden mit einer Reduktion des Verantwortungsbewusstseins gegenüber Dingen, die sich ausserhalb der Siedlungsgrenzen abspielen (NIMBY-Syndrom).

Tore sind für gutverdienende Bevölkerungsgruppen zum Statussymbol und Gradmesser für Einkommen und Reichtum geworden (TAKAHASH 1997). Die Kategorisierung nach Wohnadresse und Postleitzahl wird von gesellschaftlichen Randgruppen als Stigmatisierung und Zeichen der Intoleranz interpretiert. REICH (1991) sieht in dieser «Sezession der Erfolgreichen» eine Spaltung der amerikanischen Gesellschaft in zwei Klassen, welche im Gesellschaftsvertrag verankerte Werte wie Solidarität und Gemeinschaft fundamental in Frage stellt.

\subsection{Alternativen zum Wohnen in Gated Communities}

Die öffentliche Planung hat es bislang weitgehend versäumt, auf lokaler und staatlicher Ebene Alternativen zu Gated Communities zu entwickeln und die Bevölkerung im Sinne der Eigenverantwortung vermehrt an der Umsetzung neuer, bewohnerorientierter Entwicklungskonzepte zu beteiligen. Anstatt wie in segregierten Wohngebieten auf architektonische «Verteidigungselemente» zu setzen und in klar voneinander getrennten Nutzungsdimensionen zu denken, könnten Architekten und Raumplaner versuchen, ganzheitliche Siedlungen mit einer Vielfalt verschiedener Nutzungsformen zu entwickeln, welche für Bewohner Anreize schaffen, eine Gemeinschaft aufzubauen und miteinander zu interagieren. Wenn sich Menschen mit ihrem räumlichen Umfeld identifizieren («Territoriality»), mit ihren Mitbewohnern solidarisieren («Natural Surveillance») und Pflichtbewusstsein zeigen, wird ein übergeordneter «Defensible Space» erzeugt, der effizienter gegen Kriminalität schützt als Mauern und Tore, die den Bewohnern die Verantwortung für das Geschehen auf der Strasse abnehmen und eine wirksame soziale Kontrolle unterbinden. Lebenswerte und sichere Wohnviertel sind auch ohne radikale Absperrmassnahmen wie in Gated Communities realisierbar. Seitens der Neo-Traditionalisten (vgl. z.B. Calthorpe 1993) gibt es - angefangen von Verbesserungsvorschlägen für das Strassenverkehrsnetz einzelner Stadtviertel bis zu regionalen Revitali- sierungsplänen - zahlreiche Anregungen zur Diversifizierung der Siedlungsstrukturen. Konzepte wie Transit-Oriented Developments, Pedestrian Pockets oder Traditional Neighborhood Developments versuchen, das verlorengegangene Gleichgewicht zwischen privaten und öffentlichen Räumen durch eine Erhöhung der Nutzungsvielfalt wiederherzustellen. Auch wenn bei der Umsetzung im Einzelnen verschiedene Aspekte im Vordergrund stehen, orientieren sich diese Konzepte an folgenden Grundprinzipien: Auf regionaler Ebene bietet sich eine Konzentration des Siedlungswachstums entlang von Transitverkehrsachsen an. Das nicht zuletzt bei privatisierten, ummauerten Wohnenklaven allgegenwärtige Landnutzungsmuster des Single Use Zoning könnte durch Planungsrichtlinien und Standards für eine fussgängerfreundliche Gestaltung und Nutzungsdurchmischung ersetzt werden, verbunden mit einer stärkeren Gewichtung des öffentlichen Raumes.

Erst wenn Gated Communities als eine in vielen Fällen durchaus verständliche Antwort auf verschiedenste gesellschaftliche, politische und wirtschaftliche Problemkreise wahrgenommen werden, ist ein erster Schritt in die richtige Richtung getan. Denn nach wie vor betrachten eine breite (amerikanische) Öffentlichkeit und sogar Fachleute der Planung Gated Communities viel zu oft als ein selbstverständliches Element des gebauten Raumes, das nicht weiter kritisch hinterfragt zu werden braucht.

\section{Literatur}

Alexander, G. (1994): Conditions of «Voice»: Passivity, Disappointment and Democracy in Homeowner Associations. - In: Barton, S.E. \& C.J. Silverman (Hrsg.): Common Interest Communities: Private Governments and the Public Interest. - Berkeley: Institute of Governmental Studies Press: 145-168.

Atteslander, P. (1995): Methoden der empirischen Sozialforschung. - Berlin: Walter de Gruyter.

Banham, R. (1987): Los Angeles. The Architecture of Four Ecologies. - London: Penguin Books.

BLAKELY, E.J. \& M.G. SNYDER (1997): Fortress America. Gated Communities in the United States. - Washington D.C: Brookings Institution Press.

Bucher, K. (1998): Gated Communities: Eine Vergleichsstudie privatisierter Wohngebiete in Südkalifornien. - Alterssiedlungen als Gated Communities. Sicherheit und politischer Einfluss von Alterssiedlungen. - Lizentiatsarbeit, Geographisches Institut der Universität Basel, Abt. Humangeographie/Stadt- und Regionalforschung.

Calthorpe, P. (1993): The Next American Metropolis. Ecology, Community, and the American Dream.-New York: Princeton Architectural Press.

Chase, J. (1997): My Urban History: Paranoia Inform- 
ing Place Making. - In: ElLIN, N. (Hrsg.): Architecture of Fear. - New York: Princeton Architectural Press: 159-185.

COHEN-EDWARDs,P.(2000):Manhattan Beach-Prestigious Gated Community.-http://www.manhattanvillage.com/, 02.08.2000.

Community Associations Institute Research FounDATION (2000): National Survey of Homeowner Satisfaction. - http://www.cairf.org/research/gallup-1.html, 02.08.2000.

Cullingworth, B.J. (1993): The Political Culture of Planning: American Land Use Planning in Comparative Perspective. - London: Routledge.

Dunbar, P.M. (1988): The Homeowners Association Manual. - Tallahassee: Suncoast Professional Publishing Corporation.

Dychtwald, K. \& J. Flower (1990): Age Wave. The Challenges and Opportunities of an Aging America. New York: Bantam Books.

Foldvary, F. (1994): Public Goods and Private Communities. The Market Provision of Social Services. Aldershot: Edward Elgar.

FrEY, B.S. (1997): Unerwünschte Projekte, Kompensation und Akzeptanz. - In: Analyse \& Kritik 19 (1997): 3-14.

Glionna, J.M. (1994): Hidden Hills likes its Politics out of View. - In: Los Angeles Times, 11.4.1994, Los Angeles: A1.

GMÜNDER, M. (1998): Gated Communities: Eine Vergleichsstudie privatisierter Wohngebiete in Südkalifornien - Familienkommunen mit unterschiedlichem Status. Raumbezogener Autonomievergleich zweier Familienkommunen. - Lizentiatsarbeit, Geographisches Institut der Universität Basel, Abt. Humangeographie/Stadt- und Regionalforschung.

Grillon, N. (1998): Gated Communities: Eine Vergleichsstudie privatisierter Wohngebiete in Südkalifornien - Gated Communities als Ferien- und Zweitwohnsitz. - Lizentiatsarbeit, Geographisches Institut der Universität Basel, Abt. Humangeographie/Stadtund Regionalforschung.

Haldane, D. (1998): Town Hall Meeting on El Toro Draws 600. - In: Los Angeles Times, 13.5.1998, Los Angeles: A1.

Hunt, M. (1983): Retirement Communities: An American Original. - In: Journal of Housing for the Elderly.New York: Haworth Press.

KILDow, J. (1997): The Roots and Context of the Coastal Zone Management. - In: Coastal Management 25/3. - Washington D.C.: Department of Marine Affairs: 231-263.

LAmNeK, S. (1995): Qualitative Sozialforschung. Band 1: Methodologie. Band 2: Methoden und Techniken. Weinheim: Beltz Psychologie Verlags-Union.

LANGDON, P. (1994): A Better Place to Live. Reshaping the American Suburb. - Amherst: The University of Massachusetts Press.
Lichtenberger, E. (1999): Die Privatisierung des öffentlichen Raumes in den USA. - In: WebER, G. (Hrsg.): Raummuster - Planerstoff: Festschrift für Fritz Kastner zum 85. Geburtstag. - Wien: IRUB: 29-39.

Lichtenberger, E. (1998): Stadtgeographie. Band 1: Begriffe, Konzepte, Modelle, Prozesse. - Stuttgart, Leipzig: Teubner.

LW LaGuna Woods (2000): LW Laguna Woods. Resort Living California Style. - http://www.lwlagunawoods.com/ indexA.html, 02.08.2000.

McKenzie, E. (1994): Privatopia. Homeowner Associations and the Rise of Residential Private Government. - New Haven and London: Yale University Press.

Newman, O. (1997): Creating Defensible Space. Upland: DIANE Publishing Co.

Olson, M. (1969): The Principle of «Fiscal Equivalence»: The Division of Responsibilities among Different Levels of Government. - In: American Economic Review 59: 479-487.

Patton, M.Q. (1990): Qualitative Evaluation and Research Methods. - Newbury Park: Sage.

REICH, R.B: (1991): The Work of Nations. Preparing

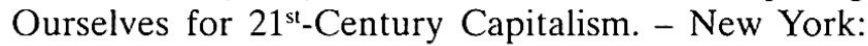
Vintage Books.

Schaltegger, S. \& M. Gmünder (1999): Private Kommunen - Funktionsweise und Entwicklung in der Praxis. - In: Aussenwirtschaft. Schweizerische Zeitschrift für internationale Wirtschaftsbeziehungen, 54. Jahrgang, Heft II: 209-224.

TAKAHASHI, L. (1997): The Socio-Spatial Stigmatization of Homelessness and HIV/AIDS: Towards an Explanation of the NIMBY Syndrome. - In: Journal for Social Science and Medicine 6: 903-914.

TREESE, C.J. (1999): Community Associations Factbook. - Alexandria: Community Associations Institute.

VIERCK, E. (1990): Fact Book on Aging. - Santa Barbara: ABC-CLIO.

Warren, R.L. (1978): The Community in America. Lanham: University of America Press.

Wehrheim, J. (1999): Gated Communities. Sicherheit und Separation in den USA. - In: RaumPlanung 87: 248-253.

Wekerle, G.R. \& C. Whitzman (1995): Safe Cities. Guidelines for Planning, Design, and Management. New York: Van Nostrand Reinhold.

\section{Zusammenfassung: Gated Communities - Ein Vergleich privatisierter Wohnsiedlungen in Südkalifornien}

Gated Communities stellen eine Extremform der Privatisierung öffentlicher Räume dar, verbunden mit einer radikalen Veränderung der Stadtlandschaft. Insbesondere in Räumen wie Südkalifornien ist eine hohe Konzentration dieser Wohnform auszumachen. Dabei lassen sich anhand umzäunter Alters- oder Feriensiedlungen sowie familienorientierter Gated Communities unterschiedliche Entwicklungsmuster identifizie- 
ren. Gründe für den Erfolg von Gated Communities sind unter anderem der Sicherheitsaspekt, die starke Identifikation mit dem Wohnumfeld sowie eine Organisationsstruktur, welche sich in ausgeprägter Form an den Präferenzen der Bewohner orientiert. Die grössten Vorteile von Gated Communities gegenüber konventionellen Siedlungen liegen bei den auf Effizienz ausgerichteten siedlungsinternen Strukturen. Demgegenüber bestehen jedoch bedeutende räumliche Probleme im Zusammenhang mit der Segregation und der Fragmentierung der Stadtlandschaft. Eine Alternative zu Gated Communities insbesondere im Hinblick auf den Sicherheitsaspekt könnten siedlungsplanerische Konzepte wie Pedestrian Pockets oder Traditional Neighborhood Developments darstellen.

\section{Summary: Gated Communities - A comparison of pri- vatised settlements in Southern California}

Gated communities represent an extreme form of privatisation of public spaces, radically changing the urban landscape. Especially areas like Southern California are distinguished by high concentrations of this kind of settlement. Gated retirement communities, second residences and family oriented gated communities create different development patterns. The secret of their success among others founds on the security asset, the strong identification with the individual living environment as well as an administrative structure that primarily focuses on the residents' interests. The biggest advantage compared to conventional settlements lies in their internal structure providing utmost efficiency. Gated communities, however, also intensify the already ongoing segregation and fragmentation of the urban landscape. Planning concepts such as «pedestrian pockets» or «traditional neighborhood developments» could be an alternative as far as the security aspect is concerned.

Résumé: Gated Communities - Comparaison de quartiers résidentiels privatisés en Californie du Sud

Les Gated Communities représentent une forme extrême de privatisation de l'espace public, menant à un changement radical du paysage urbain. On constate une concentration élevée de cette forme d'habitat spécialement dans des régions du type de la Californie du Sud. Des zones d'habitats entourées de murs ou clôtures spécialisées pour les personnes âgées, les vacanciers ainsi que les familles se développent selon des modèles différents. Le succès des Gated Communities se base entre autres sur l'aspect de la sécurité, la forte identification avec l'entourage individuel ainsi que la structure d'organisation qui s'oriente surtout sur les intérêts des habitants. Le plus grand atout des Gated Communities par rapport aux habitats conventionnels est leur structure interne visant l'efficacité. Par contre, il existe d'importants problèmes spatiaux concernant la ségrégation et la fragmentation du paysage urbain. Des projects d'habitats comme les Pedestrian Pockets et les Traditional Neighborhood Developments seraient une alternative aux Gated Communities en ce qui concerne la sécurité.

lic.phil. Markus Gmünder, Wirtschaftswissenschaftliches Zentrum (WWZ) der Universität Basel, Petersgraben 51, CH-4003 Basel.

e-mail: Markus.Gmuender@unibas.ch

lic.phil. Nathalie Grillon, Europainstitut der Universität Basel, Gellertstrasse 27, CH-4020 Basel.

e-mail:n.grillon@unibas.ch

lic.phil. Kuno Bucher, Statistisches Amt des Kantons Basel-Stadt, Webergasse 34, CH-4005 Basel.

e-mail: Kuno.Bucher@bs.ch 